\title{
¿Disrupción en la educación para la sostenibilidad?
}

\section{Disruption in education for sustainability?}

Recibido: $15 / 07 / 2013$

Revisado: 08/08/2013

Aceptado: 28/10/2013

\author{
Enric Pol y Angela Castrechini \\ Universitat de Barcelona, Espańa
}

Correspondencia: Enric Pol. Facultad de Psicología. Universitat de Barcelona. Pg. de la Vall d’Hebrón, 171. 08035. Barcelona (Spain).E-mail: epol@ub.edu

Reconocimientos: Este estudio se realizó como contribución del Grupo de Investigación PsicoSAO (2009SGR210) de la Universidad de Barcelona, reconocido por la AGAUR (Gobierno de Cataluña) a la Red de Investigación en Educación para la Sostenibilidad (www.edusost.cat), promocionado por el Departamento de Medio Ambiente de la Generalitat de Catalunya (Gobierno Catalán). Queremos manifestar nuestro agradecimiento a las personas que han colaborado en las distintas fases de la investigación, especialmente a Montserrat Écija, Montse Mora, Eric Fernández, Daniel Carro y Amaya Pou.

\section{Abstract}

This paper presents and confirms the suspicion that there is a disruption in environmental education and environmentally responsible behaviors. Children are well aware of the behaviors and values considered environmentally friendly. However, there is a statistically significant decrease in the scores during adolescence. Scores recover in the College sample but they fail to reach children's scores. This has to do more with specific characteristics of adolescence and social context than with environmental education. A questionnaire built ad hoc was applied to a sample of 2037 persons between 9 and 35 years old. It should be noted, however, that the low scores of teenagers are also notably high in the range of the used scale.

Key words: environmental education, sustainability, childhood, youth, university with nature

\section{Resumen}

El presente artículo plantea y confirma la sospecha de que existe una "disrupción" en la educación ambiental y la conducta ecológica responsable. Los niños conocen perfectamente cuáles son las conductas y valores considerados ambientalmente "correctos", sin embargo, en la adolescencia se produce un descenso estadísticamente significativo, que se recupera en la muestra universitaria, pero sin alcanzar las puntuaciones de los niños. Ello tiene más que ver con características propias de la adolescencia y el contexto social, que con la Educación Ambiental. Se aplicó un cuestionario ad hoc, a una muestra de 2037 personas entre 9 años y 35 años. Cabe destacar, sin embargo, que también las puntuaciones bajas de los adolescentes son notablemente elevadas en el rango de la escala utilizada.

Palabras clave: educación ambiental, sostenibilidad, infancia, adolescencia, universidad 
No hace muchos años, el gran reto de lo que genéricamente se llamaba Educación Ambiental (que en el campo de la psicología ambiental se focalizaba -y se focaliza- en el eje valores, creencias, actitudes y comportamientos) era el incremento de la "conciencia ambiental" (environmental concern) en los ciudadanos. Ello llevó a la creación de un gran número de escalas y cuestionarios que buscaban medir esta variable. La aplicación de algunas escalas estandarizadas (como, por ejemplo, la NEP de Dunlap y Van Liere, 1978) a poblaciones más o menos representativas de distintos países, buscaba establecer comparaciones de la evolución del ambientalismo en los diferentes lugares del mundo. En las posteriores revisiones de su propuesta, Dunlap y Van Liere (véase Dunlap, 2008; Dunlap, Van Liere, Merting \& Jones, 2000), al igual que Stern (1992), advierten de la dificultad de medición de actitudes ambientales, que se ve aún más complicada por la profusión de instrumentos concebidos desde visiones nada similares y mesurando dimensiones distintas. Además, Dunlap et al. (2000) apuntan que el efecto de la deseabilidad social sesga sus resultados hacia unos resultados aparentemente positivos.

En las últimas décadas, especialmente después de la Cumbre de Río 92, la presión mediática y los programas formativos en educación ambiental (en adelante EA) se generalizaron -por lo menos en Europa-y empieza a resultar difícil que los jóvenes actuales no hayan participado en algún tipo de EA. Sin embargo, ello no parece materializarse en una conducta ecológica responsable (en adelante CER) efectiva. Uzzell (2004) ya apuntaba algunas razones para este aparente fracaso. Es especialmente destacable lo que llamó "hipermetropía ambiental", o la tendencia a preocuparse por los grandes problemas ambientales globales, pero no atender -o incluso minimizar- las implicaciones ambientales inmediatas de las conductas y acciones cotidianas y próximas, a pesar de los bien intencionados programas de $\mathrm{EA}$, que a veces presentan efectos contrarios a los deseados. Argumenta, además, la necesidad de que la EA potencie una identidad global compartida, que incluya el respeto como uno de sus valores. Pero en los últimos años, especialmente después del inicio de la crisis económica en Europa (año 2008), hay indicios de un retroceso en la consciencia y el comportamiento pro-ambiental. Tales cambios pueden ser explicados por cambios estructurales (como por ejemplo, recortes en presupuestos destinados a acciones ambientales) y por cambios en los énfasis de la comunicación ambiental (Pol, Castrechini, Carmona, Ramírez \& Manolov, en prensa).

\section{La educación para la sostenibilidad en la infancia y la juventud}

En la literatura se observa el uso de dos etiquetas diferentes para hacer referencia al mismo fenómeno: "educación ambiental" y "educación para la sostenibilidad". La primera es más común en las primeras iniciativas o desarrollos en este ámbito, como término dominante hasta la década de los 90, mientras que la segunda es una etiqueta más frecuente en la actualidad. El cambio de uso del término "ambiental" por el de "Sostenibilidad" se dio buscando una connotación más amplia e inclusiva de su significado (aspectos ambientales, sociales, económicos, etc.), desde su lanzamiento en el Informe Brundtland en 1987. Sin embargo, en los últimos años se está restringiendo cada vez más a su dimensión económica. El año 2005 aparece como clave. En ese año se inició el Decenio de la Educación para el Desarrollo Sostenible, instituido por Naciones Unidas (Resolución 57/254) como un llamamiento a los educadores de todas las áreas y niveles, tanto de la educación formal (desde la Escuela Primaria a la Universidad) como informal para contribuir a la formación de ciudadanas y ciudadanos conscientes de los problemas socioambientales (Gutiérrez et al., 2006; Vilches et al., 2009).

De acuerdo con Moyano, Encina y Vicente (2007) las investigaciones que evalúan los impactos de la educación ambiental, parecen confirmar que la EA "mejora la calidad de la educación en general, el rendimiento académico y las habilidades de ciudadanía", es decir, tienen un efecto positivo sobre el proceso de aprendizaje en sí. Sin embargo, la mayoría de los programas educativos ambientales están caracterizados por proporcionar una gran cantidad de conocimientos científicos, acerca de los problemas ambientales así como sus causas y efectos sobre el bienestar de la sociedad, más que sobre procesos formativos (Jensen \& Schnack, 2006; Tsevreni, 2011). De hecho, pocos se centran en la acción, esto es, en utilizar metodologías que fomenten el conocimiento a través de la exploración, el contacto directo con las problemáticas asociadas y que estimulen el análisis y la reflexión crítica acerca de su entorno (Breitin et al., 2009; Hart, 1997, 2003; Jensen, 1997, 2004). De acuerdo con Huckle (1991), la red ACEU (Ambientalización Curricular de la Educación Universitaria, 2003) y Mayer (2007), la educación ambiental debería incluir las características de la pedagogía crítica basada en el aprendizaje activo y experiencial, así como en la reflexión crítica. En particular algunos autores afirman 
que se debería estimular a los niños para que expresen y comuniquen sus experiencias, ideas y emociones acerca de su entorno y su vida diaria (Barratt Hacking, Barrat \& Scott, 2007). En la misma línea, Tsevreni (2011) afirma que la educación ambiental basada en el paradigma crítico debería centrarse en el empoderamiento y la acción y no sólo en la transferencia del conocimiento científico sobre la crisis ecológica, (que por otro lado se han tornado más matizados y complejos, y por tanto, menos evidentes y menos fáciles de comunicar y/o transmitir).

Por otra parte, Davis (2009) distingue tres tipologías de investigaciones sobre la educación ambiental en relación con la infancia: (i) educación en el medio ambiente, las cuales se centran en explorar las relaciones que establecen los niños con la naturaleza; (ii) la educación sobre el medioambiente, son los estudios que exploran la comprensión que tienen los niños sobre los diferentes temas ambientales; y (iii) la educación para el medio ambiente, hace referencia a las investigaciones que se centran en los niños como agentes de cambio. Cada uno de ellos representa un tipo diferente de énfasis: en las relaciones, la concepción o la acción. En una revisión de la literatura, esta investigadora concluyó que la mayoría de las publicaciones tratan sobre la primera tipología de estudios, observándose pocos registros de las otras dos tipologías. La pregunta que cabría formularse es sí esta diferencia se debe a la baja presencia de programas educativos enfocados hacia las dos últimas tipologías.

Adicionalmente, no hay que perder de vista la importancia del contexto cultural en la educación para la sostenibilidad. Existen factores estructurales que posibilitan o dificultan las prácticas respetuosas con el medio ambiente. Sandoval (2012) insiste en que la EA se debe entender "como un proceso que ocurre en diferentes espacios sociales más allá de las Instituciones Escolares” (p. 181). La autora sugiere ampliar la noción de medio ambiente que subyace a la EA clásica e incluir como objetivo de trabajo el desarrollar patrones de comportamiento respetuosos con el entorno partiendo desde el análisis de las prácticas culturales argumentando que ésta es la manera de garantizar la concordancia entre los comportamientos que se enseñan y los que se practican. En esta misma línea, Guevara (2002) ante la formulación de proyectos de intervención ambiental comunitaria (que es otra vía de educación ambiental) enfatiza la necesidad de "conocer los patrones comportamentales, los hábitos y costumbres de la población [...] para generar un cambio en ellos" (p. 278).

\section{La EA desde la Psicología}

La importancia de la implicación ambiental de los niños y jóvenes ha sido reconocida como un factor clave para cultivar una disposición de cuidado hacia el medio ambiente a lo largo de la vida (Davis, 2009; Duhn, 2012) Sin embargo, es escasa la literatura psicológica que incluya activamente las peculiaridades de los diferentes estadios del ciclo de vida humano, especialmente de la infancia y adolescencia.

Las contribuciones de la psicología, se centran más sobre la construcción de actitudes (Moyano et al., 2007; Páramo \& Gómez, 1997) y normas sociales, a veces desde la información, los valores y las creencias (Pinheiro y Pinheiro, 2007); otras veces sobre normas sociales, o sobre engagement, como se puede seguir en Kaiser y Byrka (2011).

Quizás las cuestiones que la psicología ambiental no ha tomado suficientemente en consideración, cuando se ocupa de la formación de valores, actitudes y comportamientos para aplicarlos a la educación, son las características propias de los estadios de desarrollo del niño (Fraijo Sing, CorralVerdugo, Tapia \& García, 2012). Las aportaciones de la psicología del desarrollo, desde las contribuciones más clásicas de Piaget, Bruner o Vygostki permiten entender parte del fenómeno, como en su momento trataron de hacer Muntañola (1980), Spencer y Darvizeh (1981), entre muchos otros en las décadas de los setentas y los ochentas del S. XX.

Para estos autores, los niños entre los 9-11 años se hallan en la etapa de las operaciones concretas y desarrollan la de las operaciones abstractas a partir de los 11-12 años. Aún así, esta interpretación - aplicada a la educación ambiental según Spencer y Darvizeh (1981) - llevó a una considerable infravaloración de las destrezas y el potencial ambiental de poseen los niños de más corta edad, y a su vez, una cierta desconsideración de las características más conflictivas de la adolescencia y sus crisis. Como señala Funes (2010), a pesar que para su maduración el adolescente requiere diferenciación (que a veces supone confrontación, oposición a la norma y conflicto), hay que seguir aportándole apoyo positivo. Si lo trasladamos al contexto de lo ambiental, cuando lo ambiental y sostenible se ha convertido en un mensaje que se asocia con la estructura social dominante (situación frecuente en Europa), puede ser rechazado o menospreciado, como forma de diferenciación, lo cual explicaría parte de los comportamientos y actitudes poco 
claras de los adolescentes en relación a la cuestión ambiental. Recientemente parecen estar de nuevo en auge los estudios que exploran las destrezas de los niños de corta edad. Así, Evans, Juen, Corral-Verdugo, Corraliza y Kaiser (2007) compararon las actitudes y el compromiso de niños de entre 4 a 6 años de cuatro países: USA, Austria, México y España no encontrando diferencias significativas dentro de este rango de edad. A pesar de ello, el estudio resulta relevante por la franja de edad que exploran.

Guevaray Fernández (2010) publicaron una investigación en Puebla en la que se comparaba el nivel de conocimientos ambientales en nińos de primaria entre 1994 y 2006. En general encontraron un muy bajo conocimiento de los temas ambientales. A pesar de este bajo nivel de conocimiento, se observó un ligero aumento en el período de estudio.

En el contexto europeo, en un estudio realizado por Grønhoj y Thøgersen (2009) se encontró que los adolescentes están menos ambientalmente comprometidos que sus padres, lo que estos autores llaman el "gap generacional". Encontraron que aunque las actitudes hacia el medio ambiente en general son positivas, los valores y las conductas comparativamente son menores. Atribuyen la causa de este hecho a las barreras asociadas al estadio del desarrollo en el que se encuentran los adolescentes, en el cual los valores prioritarios de esta edad difieren de la de los adultos.

En la revisión de la literatura sobre el diseño y evaluación de programas de educación ambiental que persiguen fomentar conductas ecológicamente responsables destacan las referencias a constructos teóricos propios de la psicología social como lo son las actitudes, los conocimientos y las intenciones de conducta. Sin embargo, también se ha destacado la relevancia de otras variables entre ellas el papel de la influencia social. En concreto, Schultz, Nolan, Cialdini, Goldstein y Griskevicius (2007) destacan el poder "constructivo, destructivo y reconstructivo" de las normas sociales. Se ha comprobado que éstas proporcionan un estándar del que las personas no quieren desviarse.

Pol (2000) propone el Modelo de las Cuatro Esferas; pretende ir más allá de la asunción clásica de las tres dimensiones de las actitudes e incluye racionalidad, emoción, funcionalidad y la influencia social como esferas estrechamente interconectadas y relacionadas entre sí (ver Figura 1).

El modelo integra diferentes supuestos teóricos para explicar los cambios de actitudes y comportamientos. Asimismo, este modelo parte del supuesto que la información

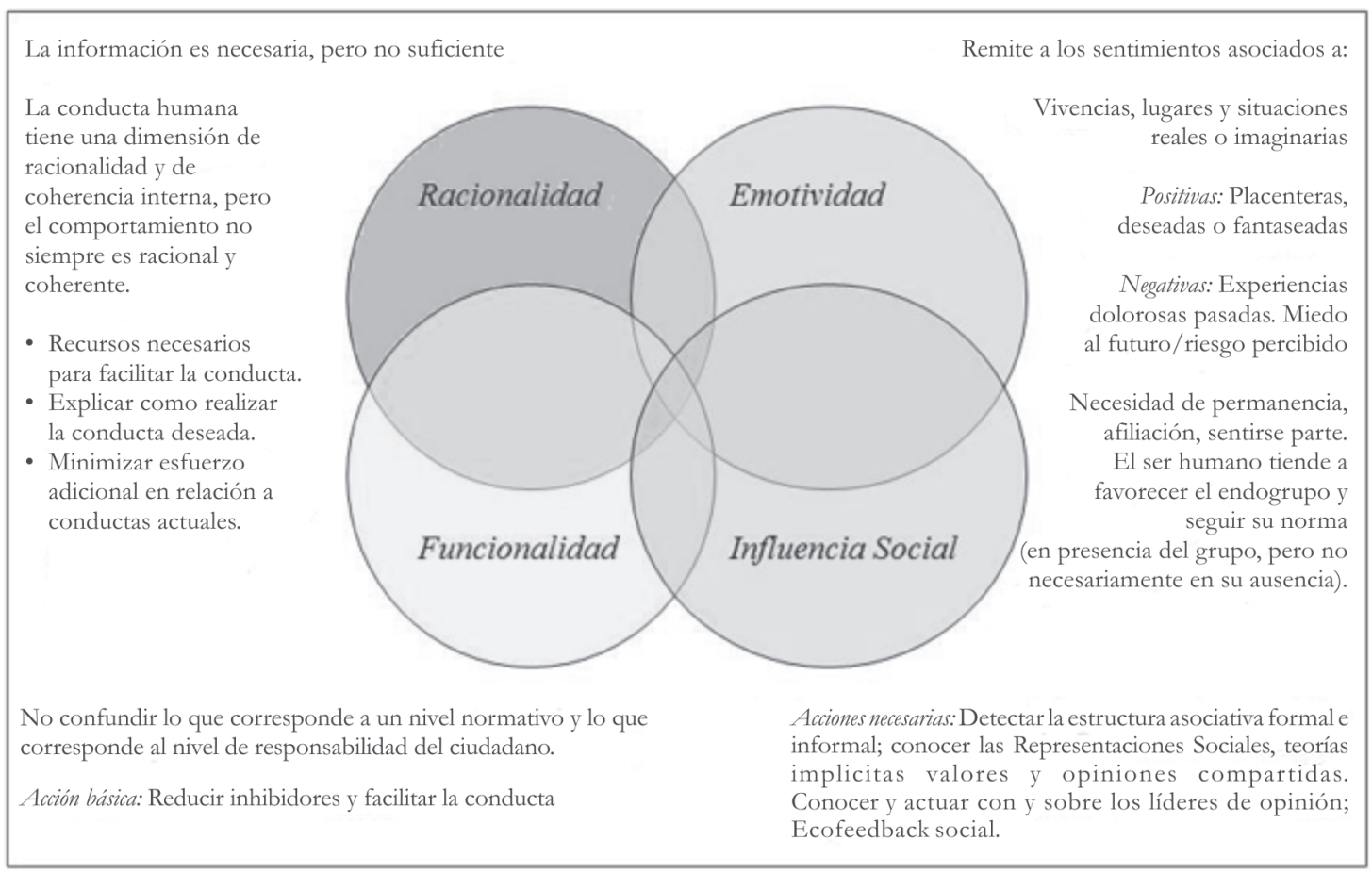

Figura 1. Modelo de las 4 Esferas (Pol, 2000) 
es necesaria, pero no suficiente para el desarrollo de conductas y actitudes; el ser humano es más racionalizador que racional, es decir, trata de reducir sus disonancias cognitivas (en la tradición de Festinger) e integra en sus esquemas cognitivos preferentemente la información que refuerza su posición (Festinger, 1957). Ello es relevante ya que puede invertir la relación de causalidad actitud-conducta por la de conducta-actitud. Pero la dimensión racional y cognitiva está siempre influenciada por la dimensión emocional, y por los mecanismos de influencia social. Además, la congruencia entre actitud y conducta depende en buena medida del "saber hacer" y del "poder hacer", es decir, tener el conocimiento del cómo y disponer de los recursos para la ejecución sin que supongan para la persona un sobreesfuerzo superior del que está dispuesto a realizar.

El modelo considera que las cuatro dimensiones están siempre activas en el comportamiento, pero puede variar el peso de cada una de ellas dependiendo de la situación específica.

\section{Contexto}

En Europa, desde los años 90 se ha ido generalizando la incorporación en los centros escolares de programas transversales de EA, con la inclusión de forma normalizada de conocimientos, valores y buenas prácticas ambientales en los currículos educativos. Sin embargo, en algunos casos esta incorporación convive con algunas acciones excepcionales, como jornadas, fiestas o semanas dedicadas a cuestiones ambientales o de sostenibilidad. Ello, no solo en la escuela sino también en las universidades, con programas de ambientalización o de sostenibilización de la universidad, no solo académicos sino también de gestión. En Cataluña conviven dos programas de "sostenibilización" de la educación: el programa "Escola Verda" (Escuela Verde, 2012) y la Agenda 21 Escolar (Barcelona City Council, 2010). Son programas de adscripción voluntaria de los centros, y comportan la adopción explícita de pautas de comportamiento e incorporación de contenidos y valores de orientación ambiental. Ambos comprenden la adopción de buenas prácticas, especialmente con la separación y minimización residuos, la incorporación de huertos escolares como formas de implicación de los niños, pero también de conocimientos en ciencias naturales y sociales en los currículos académicos. Adicionalmente, la Agenda 21 escolar incluye el involucramiento en actos cívicos junto con la administración local, así como la participación en la vida social y las cuestiones urbanas en el barrio. Ambos programas se aplican tanto en la educación primaria como en la secundaria. Sin embargo, y como relata Esteban (s/a), lo más frecuente es que exista una visión global del medio ambiente en la educación primaria, pero que la misma disminuya de forma significativa en la secundaria y el bachillerato. Afirma esta autora que "a medida que avanzan las etapas educativas, la EA encuentra serias dificultades de desarrollo" (p. 16).

A pesar que hemos mencionado el 2005 como una fecha clave, en su último giro, la Educación Ambiental cuenta con una muy larga historia. En una concepción laxa, podríamos remontarnos al mismo S XIX, y el inicio de los movimientos naturalista y conservacionista. Una concepción más estricta nos lleva a iniciativas civiles desde los años sesenta. A un nivel formalizado, debemos remontarnos por lo menos a la Conferencia de Estocolmo de 1972, y las que la siguieron, como Tiblisi 1977, Moscú 1987, Tesalónica 1997, etc. A lo largo de estos más de 40 años, el contexto, los énfasis y los promotores han ido cambiando, confiriéndole unas connotaciones sociales no solo distintas sino, incluso, contrapuestas. Pasamos de unos inicios "revolucionarios", a una situación actual en que la EA es promocionada por y desde estructuras de poder (Pol, 2000). Ello cambia el valor relativo y efectivo de los mensajes, y nos lleva a plantearnos si ¿se dan valores, creencias y comportamientos positivos hacia la sostenibilidad entre los niños y los jóvenes?, ¿tienen la misma presencia en la infancia y la juventud? En un contexto como el europeo, en que como hemos dicho, puede considerarse que todos los niños y jóvenes han estado expuestos a programas de educación ambiental, ¿su efecto positivo se rompe en algún momento del ciclo vital?, ¿por qué?. El propósito de la investigación que se presenta en el presente artículo es contribuir a dar respuesta a estas preguntas investigando la presencia de estos valores, creencias y comportamientos en el colectivo de los niños y jóvenes e indagando si existen diferencias significativas dependiendo del estadio evolutivo en el cual se encuentren.

\section{Método}

\section{Participantes}

El estudio se realizó con niños, jóvenes y adultos de edades comprendidas entre los 9 y los 35 años. La muestra final quedó constituida por 2037 personas, de las cuales el $44,7 \%$ eran personas de género masculino y $55,3 \%$ de 
género femenino. La media de edad fue de 14,91 y una desviación estándar de 5,362. La distribución geográfica no es proporcional ni representativa del territorio de Cataluña, pero a partir de la selección de los centros de estudio, se procuró abarcar a personas de diferentes tipologías geográficas y sociales. Aun así, la mayor porción de la muestra se obtuvo del Área Metropolitana de Barcelona (54,8\%).

\section{Instrumento}

Partiendo de la revisión de la literatura sobre escalas de actitudes, creencias y comportamientos ambientalmente responsables, ya existentes y contrastadas, se elaboró un cuestionario ad hoc común para los diferentes niveles de edad contemplados en el estudio.

En particular, se consultaron diversas escalas e instrumentos de medida habitualmente empleados en la medición de actitudes (Corraliza \& Martín, 1996; Corraliza \& Berenguer, 2000), creencias y valores (Bechtel, Corral-Verdugo \& Pinheiro, 1999; Dunlap \& Van Liere, 1978; Dunlap et al., 2000; Weigel \& Weigel, 1978), comportamientos (García Mira, 2006; Hernández, CorralVerdugo, Hess \& Suárez, 2002; Hess, 1996; Real \& García Mira, 2001). Se consideró que ninguna de ellas se ajustaba a los objetivos del estudio, ni a la diversidad de edades contempladas en la investigación. No obstante, de cada una de ellas se extrajeron algunos aspectos temáticos que se consideraron relevantes para la investigación.

El cuestionario se construyó siguiendo el Modelo de las 4 Esferas (Pol, 2000) y agregando una quinta dimensión de comportamientos expresados directamente. El objetivo no era crear una escala de actitudes sino incluir la presencia activa de conocimientos sobre qué se tiene que hacer y cómo hacerlo (lo que dicen las esferas de la cognición y la funcionalidad); la disposición a hacer los comportamientos deseados (lo que Fishbein y Ajzen denominan "norma subjetiva"); las emociones que desencadenan algunos temas relacionados con el medio ambiente y la sostenibilidad, a veces relacionadas con ilusiones y deseos, pero también con miedos (esfera de la emoción); la influencia que ejercen los otros en el comportamiento personal (se reirán de mí si hago o dejo de hacer, etc.), lo que indica la influencia social. En las preguntas directas sobre comportamientos ambientales concretos se recogen algunos comportamientos relacionados con residuos, energía, agua y movilidad. La atribución a priori a las dimensiones de este modelo da la agrupación de ítems de la Tabla 1.
Tabla 1

Agrupación de los ítems de acuerdo al Modelo de las 4 Esferas (Pol, 2000)

Esfera de la Cognición y la Racionalidad

2. Cuando sea grande, el medio ambiente estará tan mal que tendremos problemas para vivir

3. El agua se puede acabar si la gastamos mucho

5. La energía nuclear es más segura de lo que la gente piensa

6. No hay que preocuparse mucho por el medio ambiente, la tecnología lo solucionará

12. Tener más dinero ha de ser más importante que la protección del medio ambiente

Esfera de la Emotividad

1. Me agradaría poder llegar a todas partes en coche

4. La contaminación no tiene nada que ver conmigo

7. Me sabe mal que desaparezcan todos los osos

11. Me molesta cuando alguien lanza papeles al suelo

9. Podemos gastar toda el agua que deseemos porque es natural y siempre habrá

Esfera de la Funcionalidad

8. En casa NO separamos los residuos porque los contenedores están muy lejos.

13. En casa, NO separamos los residuos porque no tenemos espacio suficiente para poner todas las bolsas, cubos o recipientes necesarios

28. Normalmente cuando tengo calor abro la ventana sin mirar si la calefacción o la estufa está apagada

Esfera de la Influencia Social

10. En casa pensamos que separar los residuos es una tontería

15. Normalmente cuando como por la calle, lanzo el papel al suelo

17. Mis amigos se meten conmigo si tiro restos de comida al lugar del papel

19. Si no me ve nadie lanzo los papeles al suelo

21. Mis amigos se ríen de mi si busco una papelera para lanzar el papel

25. Me meto con mis amigos si lanzan papeles al lugar de los plásticos

27. En casa se separan los residuos

Comportamientos

14. Apago la TV con el mando a distancia, no por el interruptor

16. Dejo el grifo abierto cuando me lavo los dientes

18. Cuando veo a alguien que hace cosas contra el medio ambiente le llamo la atención

20. Me fijo si he dejado la luz encendida 0 apagada cuando salgo de una habitación

22. Procuro utilizar el papel por las dos caras

23. Utilizo papel reciclado

24. Hablo sobre el medio ambiente con mis amigos, familiares y/o conocidos

26. Separo el papel del vidrio y del plástico. Y lanzo cada cosa en su lugar 
Se ha de tener en cuenta que los comportamientos, los valores y las creencias no se ubican nunca en una esfera pura, sino en la intersección entre dos o más esferas. Se puso especial cuidado en controlar que la formulación de las preguntas no indujera directamente la respuesta además de lo que se denomina efecto "deseabilidad social". Se aplicaron estrategias de contrabalanceo entre preguntas opuestas y otras medidas habituales, en el pre-test del cuestionario. En una u otra dirección de respuesta, se han empleado siempre formulaciones positivas, sobre una escala de 4 puntos (4 para "máximo acuerdo", "siempre" y 1 para "nada de acuerdo", "nunca”).

\section{Procedimiento}

El cuestionario fue pilotado con una muestra de 200 personas de las diferentes franjas de edad, abarcando desde $4^{\circ}$ grado de Educación Primaria hasta el $2^{\circ}$ año de estudios universitarios. Posteriormente, el cuestionario fue aplicado en el contexto escolar/académico, tanto en centros públicos como en centros concertados ${ }^{1}$, en 8 escuelas de Educación Primaria, 8 centros de Educación Secundaria, Bachillerato y Formación Profesional. A nivel superior se aplicó en 3 universidades, escogiéndose estudiantes de diferentes carreras, tanto de "letras" como de "ciencias" y de tecnología.

\section{Análisis de resultados}

El análisis estadístico de la muestra se realizó con el programa SPSS 15.0. En primer lugar se aplicó el Alfa de Cronbach para analizar la fiabilidad del instrumento. Posteriormente se procedió a calcular las puntuaciones medias obtenidas en las dimensiones del instrumento para cada una de las franjas de edad y se realizó un Análisis de Varianza (ANOVA) para determinar la existencia de diferencias significativas entre los grupos. En tercer lugar, dado la novedad del instrumento y la construcción a partir del Modelo teórico de las 4 Esferas, se realizó un Análisis de Componentes Principales (ACP) para observar la agrupación estadística de los ítems. Por último, con el objetivo de analizar el efecto de la edad se realizó un Análisis de Varianza (ANOVA) a partir de las puntuaciones factoriales, las cuales se incluyeron en la base de datos como variables nuevas.

1 En España se entiende por centros "concertados", aquellos centros de titularidad privada que cuentan con ayudas públicas para costear parte de sus gastos.

\section{Resultados}

\section{Índice de Fiabilidad del Instrumento}

El primer paso consistió en calcular el índice de fiabilidad global, obteniéndose un Alfa de Cronbach de $\alpha=.730$, y de $\alpha=.745$ basado en los datos tipificados. De acuerdo con George y Mallery (1995) los valores obtenidos, entre .8 y .7 , permiten considerar la consistencia interna del instrumento como "aceptable".

\section{Análisis de Varianza}

A continuación, los datos se agruparon por franjas de edad en cuatro grupos: 9-13, 14-15, 16-17 y 18-en adelante y se efectuó un Análisis de Varianza (ANOVA) para las puntuaciones obtenidas en las diferentes dimensiones del cuestionario (ver Tabla 2).

Como se puede apreciar en la tabla anterior, en todos los casos se observan diferencias significativas entre las puntuaciones obtenidas por los grupos de edad. En particular, el gráfico de medianas muestra una evolución característica en forma de "U" en relación con la edad (ver Figura 2).



Figura 2. Puntuaciones globales por edades

Este resultado parece indicar que existe una "disrupción" entre la educación ambiental y la conducta ecológica responsable según el estadio del ciclo de vida de las personas. Como se puede apreciar en la Figura 2, los niños de 9 a 13 años conocen perfectamente cuáles son las conductas básicas adecuadas y expresan unos valores y creencias más que deseables desde el punto de vista ambiental y/o de la sostenibilidad. Así mismo, en la adolescencia se produce un descenso de los mencionados valores que tiende a recuperarse en la juventud y madurez, pero sin conseguir 


\begin{tabular}{|c|c|c|c|c|c|c|}
\hline & & $\begin{array}{l}\text { Suma de } \\
\text { cuadrados }\end{array}$ & GI & $\begin{array}{c}\text { Media } \\
\text { cuadrática }\end{array}$ & $F$ & Significación \\
\hline \multirow[t]{3}{*}{ Emotividad } & Entre grupos & 45.902 & 3 & 15.301 & 69.328 & .000 \\
\hline & Dentro grupos & 503.640 & 2282 & .221 & & \\
\hline & Total & 549.542 & 2285 & & & \\
\hline \multirow[t]{3}{*}{ Racionalidad } & Entre grupos & 22.286 & 3 & 7.429 & 34.535 & .000 \\
\hline & Dentro grupos & 490.668 & 2281 & .215 & & \\
\hline & Total & 512.955 & 2284 & & & \\
\hline \multirow[t]{3}{*}{ Influencia } & Entre grupos & 21.321 & 3 & 7.107 & 36.308 & .000 \\
\hline & Dentro grupos & 446.688 & 2282 & .196 & & \\
\hline & Total & 468.009 & 2285 & & & \\
\hline \multirow[t]{3}{*}{ Funcionalidad } & Entre grupos & 14.745 & 3 & 4.915 & 11.086 & .000 \\
\hline & Dentro grupos & 1010.877 & 2280 & .443 & & \\
\hline & Total & 1025.622 & 2283 & & & \\
\hline \multirow[t]{3}{*}{ Comportamientos } & Entre grupos & 51.936 & 3 & 17.312 & 87.684 & .000 \\
\hline & Dentro grupos & 450.351 & 2281 & .197 & & \\
\hline & Total & 502.287 & 2284 & & & \\
\hline
\end{tabular}

las elevadas puntuaciones de los niños. Cabe destacar, de todas formas, que también las puntuaciones bajas de los adolescentes son notablemente elevadas en el rango de la escala utilizada

\section{Análisis de Componentes Principales}

Por otra parte, teniendo en cuenta el índice de fiabilidad obtenido en el cuestionario, construido a partir del Modelo teórico de las 4 Esferas, y teniendo en cuenta el supuesto teórico de que las actitudes y los comportamientos no se sitúan casi nunca en una sola esfera pura, sino en sus intersecciones, se trataba de encontrar dónde se situaban estas interacciones. Se realizó un Análisis de Componentes Principales (ACP) sobre el conjunto de los datos, con el objetivo de intentar obtener agrupaciones de ítems que al mismo tiempo fueran estadísticamente significativos y teóricamente coherentes. La mejor solución factorial se obtuvo con una solución forzada a 5 componentes, que en conjunto explican un $41,93 \%$ de la varianza total de la muestra (ver Tabla 3).

Aunque la varianza total es baja, aparecen algunos matices en los factores resultantes que vale la pena considerar:
1. Componente 1: Está dominado por una dimensión de funcionalidad, asociada a los residuos, con cierta carga de emotividad.

2. Componente 2: Se sitúa claramente en la intersección entre cognición y emoción, que es el campo donde se sitúan las creencias, que siempre tienen una dimensión de información, conocimiento, racionalización y emoción.

3. Componente 3: Aparece como un factor complejo, difícil de caracterizar, que parece recoger valores asociados con el civismo. Tiene un componente de emoción asociado a algunos comportamientos deseables o rechazables.

4. Componente 4: Este es un factor que, pese a no agrupar los ítems atribuidos inicialmente, tiene un claro componente de influencia social y asertividad.

5. Componente 5: Agrupa comportamientos que son principalmente hábitos. Como tales, son completamente rutinarios, de difícil modificación, pero posible a partir de información y educación.

\section{Análisis de Varianza de las puntuaciones factoriales}

Con el objetivo de analizar el efecto de la edad se obtuvieron las puntuaciones factoriales y se incluyeron en la base de datos como variables nuevas. El Análisis de Varianza 


\section{Tabla 3}

Matriz de componentes rotados para el Análisis de Componentes Principales

\begin{tabular}{|c|c|c|c|c|c|}
\hline \multirow{2}{*}{ Ítems del cuestionario } & \multicolumn{5}{|c|}{ Componentes } \\
\hline & 1 & 2 & 3 & 4 & 5 \\
\hline 27. En casa se separan los residuos & .872 & & & & \\
\hline $\begin{array}{l}\text { 13. En casa, NO separamos los residuos porque no tenemos espacio suficiente para poner todas las } \\
\text { bolsas, cubos o recipientes necesarios }\end{array}$ & .792 & & & & \\
\hline 8. En casa NO separamos los residuos porque los contenedores están muy lejos. & .780 & & & & \\
\hline 26. Separo el papel del vidrio y del plástico. Y lanzo cada cosa en su lugar & .780 & & & & \\
\hline 10. En casa pensamos que separar los residuos es una tontería & .632 & & & & \\
\hline \multicolumn{6}{|l|}{ 23. Utilizo papel reciclado } \\
\hline 9. Podemos gastar toda el agua que deseemos porque es natural y siempre habrá & & .595 & & & \\
\hline 3. El agua se puede acabar si la gastamos mucho & & .560 & & & \\
\hline 6. No hay que preocuparse mucho por el medio ambiente, la tecnología lo solucionará & & .526 & .317 & & \\
\hline 2. Cuando sea grande, el medio ambiente estará tan mal que tendremos problemas para vivir & & .487 & & & \\
\hline 4. La contaminación no tiene nada que ver conmigo & & .461 & & & \\
\hline 5. La energía nuclear es más segura de lo que la gente piensa & & .450 & & & \\
\hline 7. Me sabe mal que desaparezcan todos los osos & & .427 & & & \\
\hline 15. Normalmente cuando como por la calle, lanzo el pape al suelo & & .635 & & & \\
\hline 19. Si no me nadie lanzo los papeles al suelo & & .578 & & & \\
\hline 11. Me molesta cuando alguien lanza papeles al suelo & & .525 & & & \\
\hline 1. Me agradaría poder llegar a todas partes en coche & & .301 & .522 & & \\
\hline 12. Tener más dinero ha de ser más importante que la protección del medio ambiente & & .428 & .520 & & \\
\hline 25. Me meto con mis amigos si lanzan papeles al lugar de los plásticos & & & & .730 & \\
\hline 17. Mis amigos se meten conmigo si tiro restos de comida al lugar del papel & & & & .606 & \\
\hline 18. Cuando veo a alguien que hace cosas contra el medio ambiente le llamo la atención & & & .340 & .564 & \\
\hline 24. Hablo sobre el medio ambiente con mis amigos, familiares y/o conocidos & & & & .539 & \\
\hline 21. Mis amigos se ríen de mi si busco una papelera para lanzar el papel & & & & -.506 & \\
\hline 20. Me fijo si he dejado la luz encendida o apagada cuando salgo de una habitación & & & & & 679 \\
\hline 22. Procuro utilizar el papel por las dos caras & & & & & .580 \\
\hline 16. Dejo el grifo abierto cuando me lavo los dientes & & & & & .499 \\
\hline 28. Normalmente cuando tengo calor abro la ventana sin mirar si la calefacción o la estufa está apagada & & & & & .424 \\
\hline 14. Apago la TV con el mando a distancia, no por el interruptor & & & & & .302 \\
\hline
\end{tabular}

(ANOVA) realizado muestra diferencias estadísticamente significativas en todos los casos salvo en el último factor (ver Tabla 4).
La conjunción del comportamiento en relación a los residuos, desde una dimensión de funcionalidad (Componente 1), parece negar la hipótesis de la disrupción. La "excusa” a partir 
Pol y Castrechini

Tabla 4

ANOVA las puntuaciones factoriales obtenidas con el ACP

\begin{tabular}{|c|c|c|c|c|c|c|}
\hline & & $\begin{array}{l}\text { Suma de } \\
\text { cuadrados }\end{array}$ & GI & $\begin{array}{c}\text { Media } \\
\text { cuadrática }\end{array}$ & $F$ & Significación \\
\hline \multirow[t]{3}{*}{ Componente 1} & Entre grupos & 49.346 & 3 & 16.449 & 17.359 & .000 \\
\hline & Dentro grupos & 852.832 & 900 & .948 & & \\
\hline & Total & 902.178 & 903 & & & \\
\hline \multirow[t]{3}{*}{ Componente 2} & Entre grupos & 23.719 & 3 & 7.906 & 8.188 & .000 \\
\hline & Dentro grupos & 869.084 & 900 & .966 & & \\
\hline & Total & 892.803 & 903 & & & \\
\hline \multirow[t]{3}{*}{ Componente 3} & Entre grupos & 79.807 & 3 & 26.602 & 29.042 & .000 \\
\hline & Dentro grupos & 824.403 & 900 & .916 & & \\
\hline & Total & 904.211 & 903 & & & \\
\hline \multirow[t]{3}{*}{ Componente 4} & Entre grupos & 41.234 & 3 & 13.745 & 14.284 & .000 \\
\hline & Dentro grupos & 866.023 & 900 & .962 & & \\
\hline & Total & 907.257 & 903 & & & \\
\hline \multirow[t]{3}{*}{ Componente 5} & Entre grupos & 4.795 & 3 & 1.598 & 1.598 & .000 \\
\hline & Dentro grupos & 899.874 & 900 & 1.000 & & \\
\hline & Total & 904.669 & 903 & & & \\
\hline
\end{tabular}

de dificultadores funcionales (no tengo espacio en casa, los contenedores están muy lejos, separar residuos es una tontería....) parecen ganar peso con la edad o la responsabilidad directa de ejecución del comportamiento deseado (ver Figura 3).

En el componente 2, que está saturado por ítems que remiten a creencias y emociones, la puntuación más alta aparece en el grupo de más edad. Las creencias tienen una dimensión de cognición que se construye sobre la información, que seguramente está favorecida por la condición de universitarios de esta parte de la muestra. Habría que ver qué pasa en esta misma franja de edad en población no universitaria (ver Figura 4).

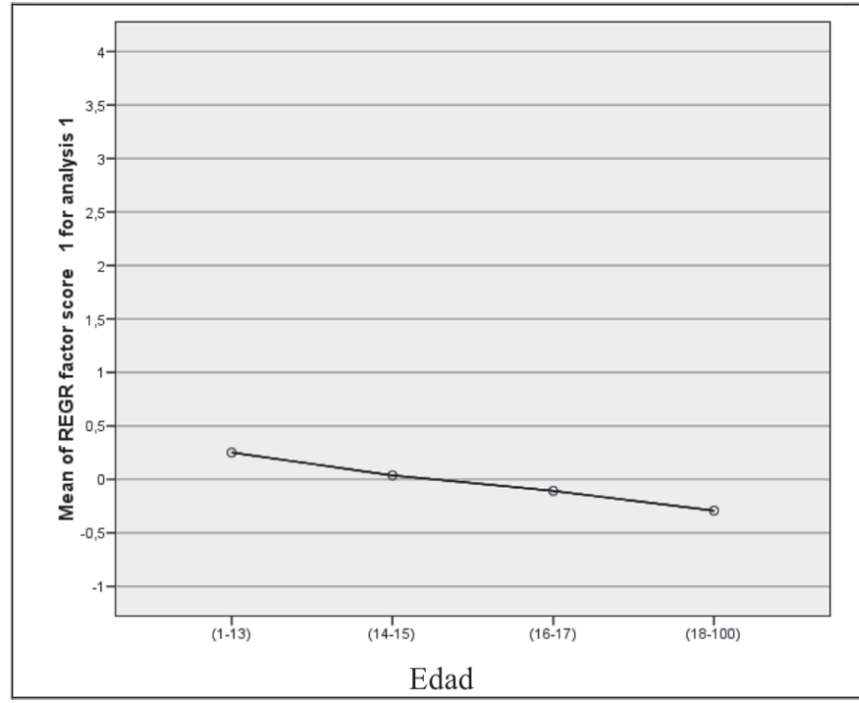

Figura 3. Medias de la puntuación factorial del Componente 1 "Residuos y Funcionalidad" para cada grupo de edad.

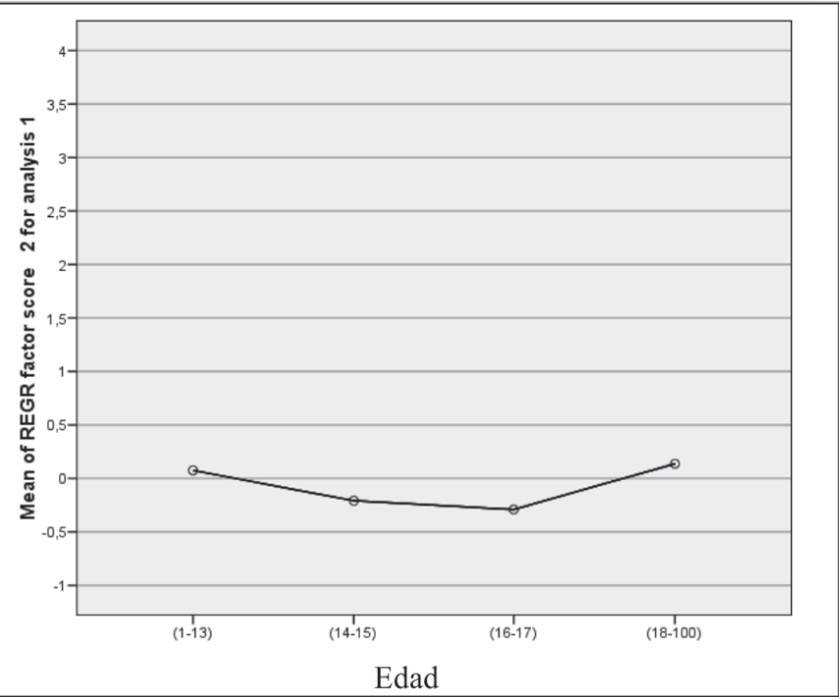

Figura 4. Medias de la puntuación factorial del Componente 2 "Creencias, cognición y emoción" para cada grupo de edad 


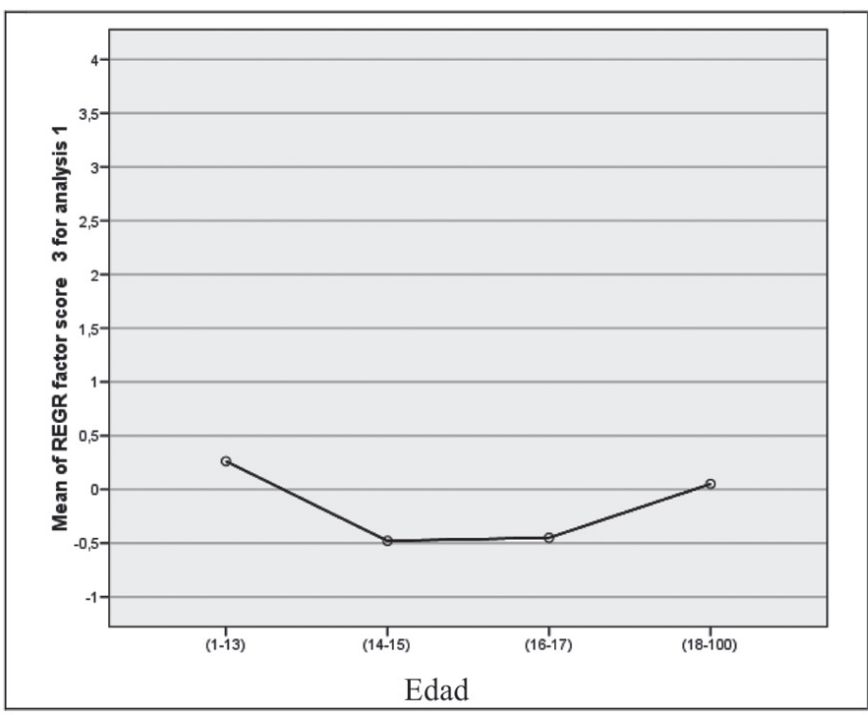

Figura 5. Medias de la puntuación factorial del Componente 3 "Civismo, emoción, transgresión" para cada grupo de edad

El factor 3 recoge conductas relacionadas con valores cívicos bastante "puros" que vienen marcados por la transgresión a veces intencional y rebelde del adolescente, o bien por fabulaciones fantásticas sobre la tecnología (como por ejemplo llegar a todas partes con coche), que parecen corregirse con la edad. En este factor, la disrupción parece pronunciarse (ver Figura 5).

La influencia social y la asertividad -componente No4- parecen estar más presentes en las franjas extremas de la muestra. En la Primaria, por estadio evolutivo del nińo, la norma es todavía "cumplir con la norma". En la Universidad, el cumplimiento de la norma de aquello correcto parece tener un peso, aunque estará en función del pensamiento social dominante en cada contexto (ver Figura 6)

En relación a los hábitos y conductas rutinarias, las puntuaciones más altas se observan en la infancia. Pareciera que la permeabilidad de la infancia hace a los niños más sensibles a la norma. Puede incidir también la recencia de la formación recibida, el mundo todavía poco matizado y dicotómico entre el bien y el mal, o lo que explicaba Piaget sobre la construcción del juicio moral en el niño. En cambio, en la población universitaria, llegado a los hábitos construidos a lo largo de más tiempo, bajo influencias de signos muy diversos, las rutinas pueden no tener mucho que ver con el pensamiento o las creencias que se manifiestan (ver Figura 7).

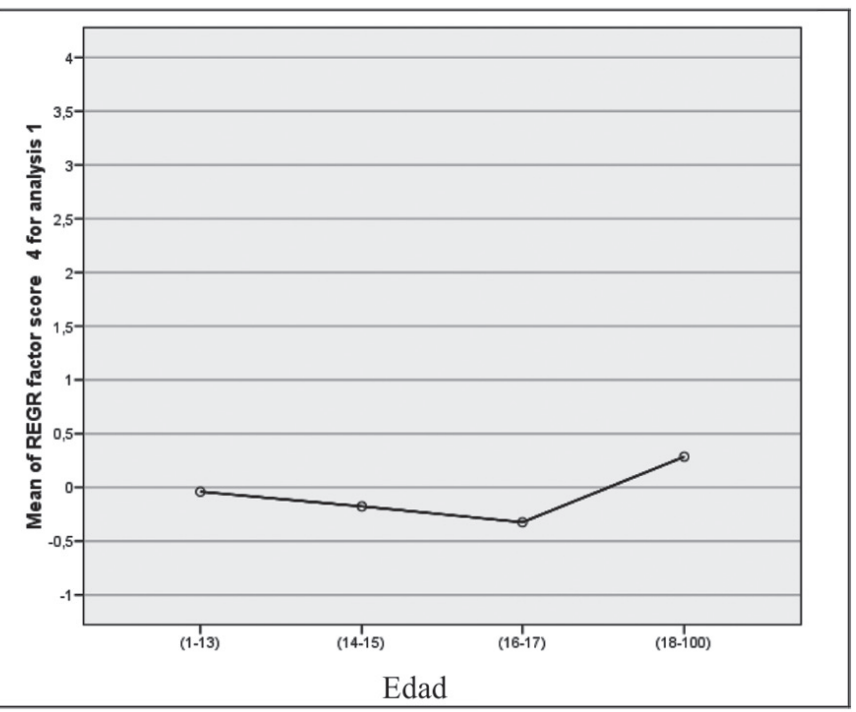

Figura 6. Medias de la puntuación factorial del Componente 4 "Influencia social y asertividad" para cada grupo

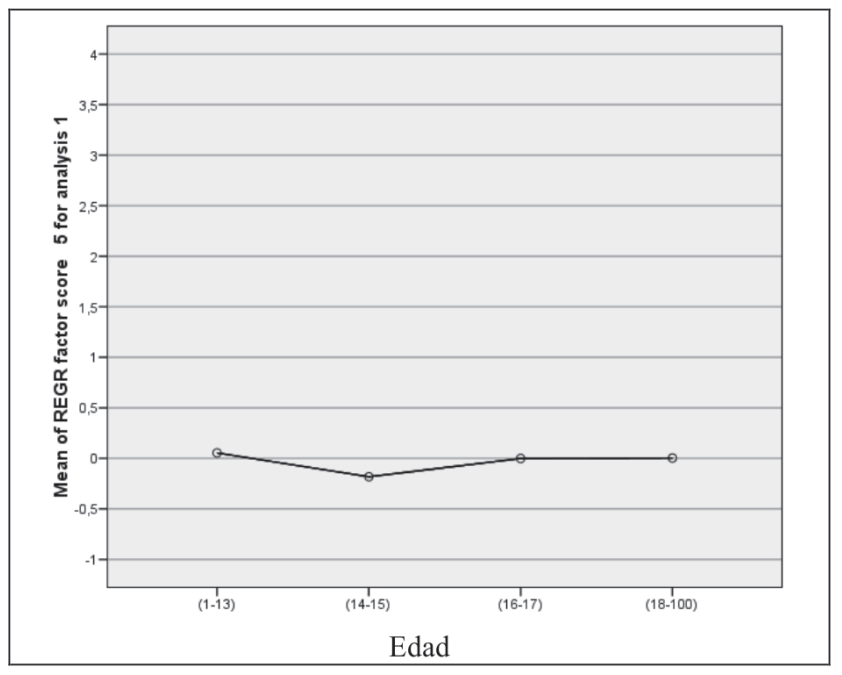

Figura 7. Medias de la puntuación factorial del Componente 5 "Hábitos y conductas rutinarias" para cada grupo de edad

Globalmente, de este análisis, podemos ver que a pesar que la disrupción está presente en diversos de los factores, e incluso parece profundizar a nivel de creencias e influencia social, cuando nos encontramos con comportamientos que dependen del esfuerzo -o del sobreesfuerzo- (factor 1) y las rutinas que se han ido estableciendo en el tiempo (factor 5), pero especialmente en la adolescencia, los comportamientos no siempre mejoran (o incluso empeoran) en relación a aquello que es deseable desde la perspectiva de la sostenibilidad. 


\section{Discusión}

Como ya hemos planteado, los objetivos principales de la investigación fueron dos, en primer lugar, estudiar si se dan valores, creencias y comportamientos propensos hacia la sostenibilidad en una muestra de niños, adolescentes y jóvenes; $y$, en segundo lugar, determinar si existen diferencias entre los diferentes grupos de edad, en particular, si la propensión hacia la sostenibilidad se rompe en algún momento del ciclo de vida, entre la infancia y la juventud. La conclusión principal de la investigación es que hay evidencias suficientes para afirmar que hay una disrupción en los valores, las creencias y los comportamientos ambientales y sostenibles al llegar la adolescencia. Estos se recuperan en parte con la edad. El contexto de la investigación pone de manifiesto que, en el caso analizado, ello no parece tan atribuible a una disminución de programas de educación ambiental en la secundaria, como interpreta Esteban (s/a), sino a características propias de la adolescencia y al contexto social externo al mundo escolar. Ello iría en la dirección que apunta Sandoval (2012) de entender la EA en relación a los espacios sociales más allá de las instituciones escolares. $Y$ estos espacios sociales tienen un amplio espectro de casuísticas, que incluyen en síla misma concepción y procesos psicológicos de la adolescencia. Así, como explica la literatura clásica sobre adolescencia, en la interpretación de Funes (2010), la diferenciación para construir su propia identidad en el adolescente, puede llevar a la confrontación y negación de la norma social. $Y$ en el caso europeo en general, y en Cataluña en particular, lo ambiental y la sostenibilidad se han asociado a la estructura social dominante, y las instituciones de gobierno son los principales emisores de mensajes sostenibilistas (no siempre coherentes con su comportamiento institucional), lo cual explicaría en parte la caída de puntuación en los adultos.

Un segundo aspecto a resaltar es que, a pesar de la disminución significativa en la adolescencia, la puntuación sigue siendo notablemente elevada. El resultado contrasta con los obtenidos por Guevara y Fernández (2010) en otro contexto social y momento histórico. Ello resalta la necesidad de tomar en consideración activa la influencia del marco socio-cultural y el contexto situacional del comportamiento y de la construcción de las actitudes.

El hecho que las mejores puntuaciones hayan sido obtenidas por la franja de edad 9 a 13 años, parece apoyar los supuestos de las teorías del desarrollo, utilizadas en su momento por Hart (1979), Muntañola 1980 y Spencer y Darvizeh (1981), como periodo en el que la norma es seguir la norma (estadio de las operaciones concretas). En este sentido, la disrupción en la puntuación de la muestra, afianza la necesidad de recuperar las especificidades psicológicas de cada estadio del ciclo de vida de las personas (también más allá de la adolescencia) para comprender el comportamiento ambiental, y si es preciso, para establecer estrategias educativas y de intervención, en la línea de lo sugerido por Fraijo et al. (2012).

De los resultados del análisis factorial, algunos resultados requieren una valoración. El componente 1 parece negar la hipótesis de la disrupción, al ser el único que no registra una recuperación después de la adolescencia. La dimensión funcional de los ítems que se agrupan en él genera la dificultad de discernir hasta donde refleja el esfuerzo o sobre-esfuerzo al que se está dispuesto a realizar o en qué medida se trata de una racionalización para reducir la disonancia cognitiva, en la línea de Festinger. En cualquier caso, parece situarse claramente en la intersección entre la esfera de la racionalidad y la de la funcionalidad.

El componente 2 es uno de los dos en que la muestra universitaria supera en puntuación al resto de los grupos. Se trata de ítems que remiten a dimensiones cognitivas y emocionales, pero en relación a aspectos que la persona percibe como que tiene poca capacidad de incidencia. Probablemente a teoría de la "hipermetropía ambiental" de Uzzell (2004) aporte una explicación plausible frente aspectos en los que hay una difícil autoatribución de responsabilidad, o si se quiere, cierta percepción de indefensión o incluso de negación del riesgo.

El sentido de transgresión adolescente de la norma que mencionábamos más arriba. Aparece claramente en el componente 3, que hemos etiquetado como de civismo, agresión y transgresión. Pero después de la crisis de la adolescencia, el componente 4 parece mostrar que es en la madurez, cuando los mecanismos de la influencia social y la asertividad alcanzan su máxima expresión. En cambio, los hábitos cotidianos (componente 5), como comportamientos rutinarios y de aprendizaje lento a lo largo del tiempo, sin excesiva intervención del pensamiento intencional, parecen ser el aspecto menos variable a lo largo de los distintos periodos. Ello puede tener una gran utilidad en el momento de establecer estrategias de educación y de intervención ambiental. 
De los datos puede concluirse que hay disrupción, pero la atribución de que las causas son distintas a las cualidades de los programas de educación ambiental requiere la realización de futuras investigaciones que permitan confirmar esta hipótesis.

Por otra parte, el estudio ha permitido detectar algunos aspectos sensibles a la intervención en educación ambiental, teniendo en cuenta tanto sus dimensiones cognitivas, afectivas y funcionales, como de influencia social. Entre los aspectos más sensibles a trabajar en la educación para la sostenibilidad, que se derivan de los datos obtenidos, destaca el diseñar programas específicos destinados a los diferentes grupos de edad y, en particular, teniendo en cuenta las prioridades en la escala de valores de los adolescentes.

\section{Referencias}

ACEU (2003) Red de Ambientalización Curricular de Estudios Universitarios. Recuperado de: http://www. edusost.cat/en/network/background

Barcelona City Council (2010) Educating for Sustainability: The Barcelona School Agenda 21 Programme. Barcelona City Council. Recuperado de: http://80.33.141.76/agenda21/documents/ EN-Barcelona\%2School\%20Agenda\%2021\%20 Programme_\%20REFESP030_10.pdf.

Barratt Hacking, E., Barratt, R. \& Scott, W. (2007). Engaging Children: Research Issues Around Participation and Environmental Learning. Environmental Education Research, 14(2), 529-544. doi: 10.1080/13504620701600271

Bechtel, R.B., Corral-Verdugo, V. y Pinheiro, J.Q. (1999). Environmental Belief Systems. United States, Brazil, and Mexico. Journal of Crosscultural Psychology, 30(1), 122-128. doi: 10.1177/0022022199030001008

Breiting, S.K., Hedegaard, F., Mogensen, K., Nielsen, \& Schnack, K. (2009). Action competence, Conflicting Interests and Environmental Education - The Muniv Programme. Copenhagen: Danish School of Education.

Corraliza, J. A. y Martín, R. (1996). Las actitudes ambientales de los españoles. Estratos, 38, 16-20.

Corraliza, J.A. y Berenguer, J.(2000). Environmental values, beliefs and actions: A situational approach. Environment and Behavior, 32(6), 832-848. doi: $10.1177 / 00139160021972829$
Davis, J. (2009) Revealing the research "hole" of early childhood education for sustainability: a preliminary survey of the literature. Environmental Education Research, 15(2), 227-241. doi: http://dx.doi. org/10.1080/13504620802710607.

Duhn, I. (2012) Making "place" for ecological sustainability in early childhood Education. Environmental Education Research, 18(1), 19-29. doi: $10.1080 / 13504622.2011 .572162$

Dunlap, R. E. (2008). The New Environmental Paradigm Scale: From marginality to worldwide use. Journal of Environmental Education, 40(1), 3-18. doi: 10.3200/ JOEE.40.1.3-18

Dunlap, R. E. y Van Liere, K. D. (1978). The "new environmental paradigm": a proponed measuring instrument and preliminary results. Journal of Environmental Education, 9, 10-19.

Dunlap, R. E., Van Liere, K. D., Merting, A. G. y Jones, R. E. (2000). Measuring endorsement of the New Ecological Paradigm: A revised NEP scale. Journal of Social Issues, 56(3), 425-442. doi: 10.1111/00224537.00176

Escuelas Verdes (2012). El Programa Escuelas Verdes. Departamento de Territorio y Sostenibilidad. Gobierno de Cataluña. Recuperado de: http://www20. gencat.cat/portal/site/mediambient/tem.718bbc757 71059204e9cac3bb0c0e1a0/?vgnextoid=4d8960add e597210VgnVCM 1000008d0c1e0aRCRD\&vgnex tchannel=4d8960adde 597210 VgnVCM 1000008d0 c1e0aRCRD\&vgnextfmt=default

Esteban, M. (s/a). La educación ambiental en Francia, Inglaterra y España. Una perspectiva comparada. Revista Iberoamericana de Educación. Recuperado de: http://www.rieoei.org/deloslectores/Macarena.PDF

Evans, G., Juen, B., Corral-Verdugo, V., Corraliza, J.A. $\&$ Kaiser, F.G. (2007). Children"s Cross-Cultural Environmental Attitudes and Self-Reported Behaviors. Children, Youth and Environments, 17(4), 128-143. Recuperado de: http://www.jstor.org/ stable/10.7721/chilyoutenvi.17.4.0128

Festinger, L. (1957). A theory of cognitive disonance. Stanford, CA: Stanford University Press.

Fraijo Sing, B.S., Corral-Verdugo, V., Tapia, C., y García, F. (2012). Adaptación y prueba de una escala de orientación hacia la sustentabilidad en niños de 
sexto año de educación básica. Revista Mexicana de Investigación Educativa, 17(55), 1091-1117.

Funes, J. (2010). Educar en la adolescencia. Nueve ideas clave. Barcelona: Graó.

García Mira, R. (2006). El asunto de la basura en Galicia. Informe para la Presidencia de la Xunta de Galicia elaborado por el Grupo de Investigación PersonaAmbiente, Universidad de la Coruña.

George, D. y Mallery, P. (1995). SPSS/PC + Step by step: A simple guide and reference. Belmont: Wadsworth Pub. Co.

Grønhoj, A. \& Thøgersen, J. (2009). Like father, like son? Intergenerational transmisión of values, attitudes and behaviors in the environmental domain. Journal of Environmental Psychology, 29, 414-421. doi: 10.1016/j.jenvp.2009.05.002

Guevara, J. (2002). Intervención comunitaria desde la Psicología Socioambiental: El caso de la basura. En J. Guevara \& S. Mercado (Coords.), Temas Selectos de Psicología Ambiental (pp. 263-290). México: FESI-UNAM, Fundación Libre, Greco.

Guevara, J. y Fernández-Crispín, A. (2010). Conocimientos y actitudes ambientales en primaria: Dos décadas de educación ambiental en México. Puebla: UPAEP.

Gutiérrez, J., Benayas, J., y Calvo, S. (2006) Educación para el desarrollo sostenible: evaluación de retos y oportunidades del decenio 2005-2014. Revista Iberoamericana de Educación, 40, 25-69.

Hart, R.A. (1997). Children"s participation: The Theory and Practice of Involving Young Citizens in Community Development and Environmental Care. London: Unicef.

Hart, P. (2003). Teacher's thinking in environmental education. Consciousness and responsibility. New York: Peter Lang.

Hernández, B., Corral-Verdugo, V., Hess, S. y Suárez, E. (2002). Los fundamentos y la estructura de la acción proecológica medidos en una escala de conductas protectoras del medio ambiente. En V. CorralVerdugo (Ed.), Conductas protectoras del ambiente. Mexico, CONACYT-Universidad de Sonora.

Hess, S. (1996). La teoría de facetas y su utilización en psicología: un estudio comparativo entre las técnicas de análisis propias de la teoría de facetas y el análisis factorial en una escala de medida de la conducta ecológica responsable. Tesis doctoral. Universidad de La Laguna
Huckle, J. (1991). Education for sustainability: Assessing pathways to the future. Australian Journal of Environmental Education, 7, 43-62.

Jensen, B. (1997). A case of two paradigms within health education. Health Education Research, 12(4), 419428. doi: 10.1093/her/12.4.419

Jensen, B.B. (2004). Environmental and health education viewed from an action-oriented perspective: A case from Denmark. Journal of Curriculum Studies, 36(4), 405-425. doi: 10.1080/0022027032000167235

Jensen, B.B., \& Schnack, K. (2006). The Action Competence Approach in Environmental Education. Environmental Education Research, 12 (3/4), 471 486. doi: 10.1080/13504620600943053

Kaiser, F.G., \& Byrka, K. (2011). Environmentalism as a Trait: Gauging people's Prosocial Personality in Terms of Environmental Engagement. International Journal of Psychology, 46(1), 71-79. doi: 10.1080/00207594.2010.516830.

Mayer, M. (2007). Cual calidad y cual formación del profesorado. Conferencia. Recuperado de: http:// www.edusost.cat/en/component/search/?searchw ord=Mayer\&ordering=\&searchphrase $=$ all

Moyano, E., Encina, Y., y Vicente, D. (2007). Evaluación del Sistema Nacional de Certificación Ambiental de Establecimientos Educacionales (SNCAE) en Chile: Operatoria e impacto. Psicología para América Latina [online]. n.10. Recuperado de: el 13 julio de 2013. En: http://www.psicolatina.org/10/evaluacion.html

Muntañola, J. (1980). La didáctica medioambiental: Fundamentos y posibilidades. Vilassar de Mar (Barcelona): Oikos-Tau.

Páramo, P., y Gómez, F. (1997). Actitudes hacia el medio ambiente: su medición a partir de la teoría de facetas. Revista Latinoamericana de Psicología, 29(2), 243-266.

Pinheiro, J.Q., y Pinheiro, T.F. (2007). Cuidado ambiental: ponte entre psicología e educaçao ambiental? Psico, 38(1), 25-34.

Pol, E. (2000) Impacte Social, Comunicació Ambiental i Participació. Barcelona: Generalitat de Catalunya, Dep Medi Ambient. Monografies Universitàries, nº 3

Pol, E., Castrechini, A., Carmona, M., Ramírez, A., \& Manolov, R. (en prensa). Communication, crise et "durabilité". Bulletin de Psyhologie. 
Real, J., y García Mira, R. (2001). Dimensiones de preocupación ambiental: una aproximación a la hipermetropía ambiental. Estudios de Psicología, 22(1), 87-96.

Sandoval, M. (2012). Comportamiento sustentable y educación ambiental: una visión desde las prácticas culturales. Revista Latinoamericana de Psicología, 44(1), 181-196.

Schultz,P.W., Nolan, J.M., Cialdini, R.B., Goldstein, N.J. \& Griskevicius, V. (2007). The Constructive, Destructive, and Reconstructive Power of Social Norms. Psychological Science, 18(5), 429-434. doi: 10.1111/j.1467-9280.2007.01917.x

Spencer, C., \& Darvizeh, Z. (1981). The case for developing a cognitive environmental psychology that does not underestimate the abilities of young children. Journal of Environmental Psychology, 1(1), 21-31. doi:10.1016/S0272-4944(81)80015-1

Stern, P.C. (1992) Psychological Dimensions of Global Environmental Change. Annual Review of
Psychology, 43, 269-302. doi: 10.1146/annurev. ps.43.020192.001413

Tsevreni, I. (2011). Towards an environmental education without scientific knowledge: an attempt to create an action model based on children"s experiences, emotions and perceptions about their environment. Environmental Education Research, 17 (1), 53-67. doi: 10.1080/13504621003637029

Uzzell, D. (2004) From local to global. A Case of Environmental Hyperopia IHDP -UPDATE. Newsletter of the International Human Dimension Program on Global Change, 04/2004: 1-2. Recuperado de: https://www.ihdp.unu.edu/file/get/7182

Vilches, A., Macías, O., y Gil, D. (2009). Década de la educación para la sostenibilidad. Temas de acción clave. Documentos de Trabajo No 1. Madrid: Centro de Altos Estudios Universitarios de la OEI.

Weigel, R.H. \& Weigel, J. (1978). Environmental concern: The development of a mesure. Environment and Behavior, 10(1),3-15. doi: 10.1177/00139165781010 
